\title{
Listening to a Painting and Communing with Bees; Heavy Metal and the Oratorio for a Million Souls
}

\author{
Dr Nigel Helyer \\ Macquarie University \\ Sydney, Australia \\ sonique1@icloud.com
}

\author{
Dr Jon Drummond \\ The University of Newcastle \\ Newcastle, Australia \\ jon.drummond@newcastle.edu.au
}

\begin{abstract}
This paper explores different approaches to the sonification and visualisation of two environmental projects undertaken by Nigel Helyer in conjunction with John Drummond.

The interactive installation Heavy Metal is focussed upon the real-time analysis and sonification of the chemical elements in a painting via a camera vision system, whilst the Oratorio for a Million Souls project concerns the behaviour and acoustic properties of live bee colonies manifest in the creation of real-time multi-channel sound compositions and associated sound architectures.
\end{abstract}

Whilst these two projects differ in terms of methodology, aesthetics and technical approach they both share a direct concern with a deep analysis of the underlying environmental structures and perceptual frameworks that emerge in direct embodied encounters - on one hand the discovery of what lies behind the surface of a painted image in terms of chemical and colour structures - and in the case of Oratorio, a compelling immersion into the acoustic environment of Bees.

Art and Science, Data sonification, Environmental sound-art, Art and Biology, Computer Graphics, Computer Vision, Data Visualisation and Analysis, Socio-Technical System, Sound Computing.

\section{INTRODUCTION AND CONTEXT FOR HEAVY METAL}

Heavy Metal ${ }^{i}$ (2016) is an art installation with an interactive camera vision system and digital audio by artist Nigel Helyer in collaboration with composer Jon Drummond and Environmental Scientist Mark Taylor. Heavy Metal was conceived and realised as part of a four-year Australian Research Council Linkage Grant project, When Science Meets Art: an environmental portrait of the Shoalhaven River Valley. A creative research collaboration between artist Nigel Helyer, environmental scientist Mark Taylor, and media theorist John Potts, in partnership with the Bundanon Trust.

The aim of the overall project is to create a complete environmental portrait of Bundanonii, a region of 1100 hectares (2700 acres) in rural New South Wales (Australia) bequeathed to the Australian people by the modernist painter Arthur Boyd ${ }^{\text {iii }}$ in 1993.
There are many ways of knowing a landscape and each approach reveals a different aspect of our relationship to the land. The varied traditional forms of landscape painting that developed in parallel with cartographic and topographic representation supplied vital economic and strategic information to expanding European societies and were a vital component of all voyages of exploration.

The advent of photography and aerial photography has transformed our view of the landscapes which we inhabit. Traditional cartographic practices are now subsumed to a large degree by views from the heavens anchored to the surface by digital survey points. Satellites probe the planetary surface in spectra beyond our own perceptual faculties, mapping such phenomena as oceanic flows, vegetation distribution and pollution plumes.

It is within this expanded context of landscape imaging that our team decided to create a representation of the terrain surrounding the Bundanon homestead that employs soil sampling as its approach. Our study of the landscape has focused directly on the stuff of the land: its soil. 
This technique can reveal natural environmental processes as well as the effects of human habitation and work sites.

The project uses a broad spectrum of techniques including environmental science, artistic practice, information technology, media technology and cultural history to develop polyvalent outcomes. Data representing environmental quality at Bundanon is digitally transformed into visual information and sound and communicated by various means: in numerous artworks; on a website $^{\text {iv }}$ devoted to the project and via GPS mapping projects for smartphone applications for mobile users on-site at the Bundanon property.

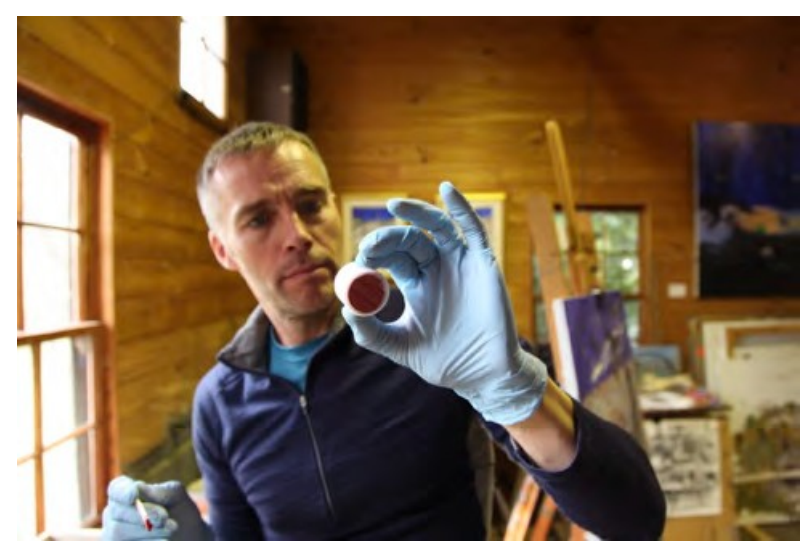

Figure 1. Dr Mark Taylor - Chemical sampling of studio paints. Image Nigel Helyer.

The environmental portrait of Bundanon also incorporates the social and cultural history of the region, as it pertains to its environmental condition. Science meets art in the communication of environmental data through artworks and media technology. Heavy Metal has been created as part of this process and invites us to interact with one of Arthur Boyd's paintings to discover a hidden world of elements and minerals in an experience that is simultaneously chemical, visual and musical.

The initial concept for Heavy $M$ etal arose after spending time on-site with Professor Mark Taylor. Mark and his students had been surveying the mineral composition of Bundanon, looking for traces of human activity that, for example, derived from farm and workshop activities, but also looking for the effects of early colonial upstream mining for gold and other heavy metals. In this extract from the Heavy Metal exhibition catalogue Nigel Helyer writes:

Elements and minerals lay buried in the landscape tracing diagrams of human activity. Specks of alluvial gold washed down to the floodplain from worked-out mountain mine shafts; existing as mineral auras that reveal the long-vanished outlines of farm buildings and the tell-tale chemical fallout from workplaces. Arthur
Boyd painted this (mineralised) landscape with colours that were themselves formulated from earthy compounds and exotic metals, milled to a fine paste in linseed oil and turpentine. ${ }^{v}$

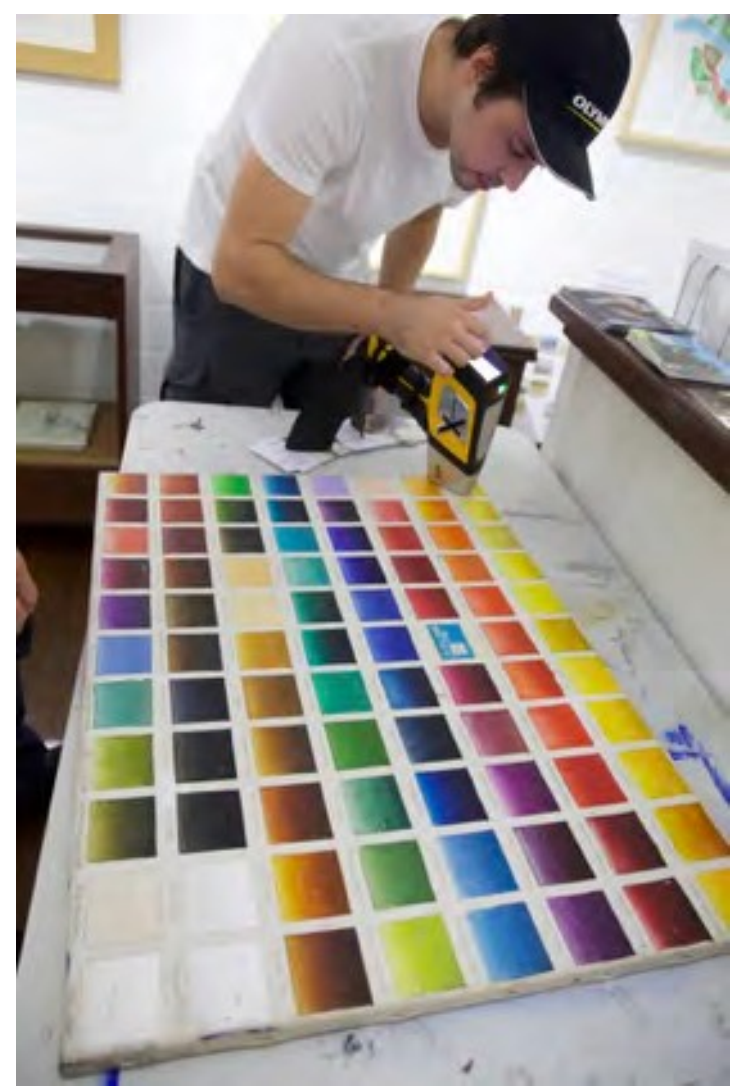

Figure 2. X-ray fluorescence sampling of studio paint chart. Image Nigel Helyer.

Our intention was to create a map of human activity based upon this forensic evidence. While we were taking environmental samples, Nigel invited Mark to visit Boyd's painting studio and bring along his portable mineral analysis machine ${ }^{\mathrm{vi}}$ as it struck him that we may have a great opportunity for rethinking Boyd's works. The starting point was that Boyd was situated in this landscape, painting the physical features and using (or making himself) colours that were substantially minerals (originally extracted from the earth), thus forming a metaphorical circuit. Mark was surprised by the massive levels of heavy metals in the materials used by painters and was keen to collaborate - so we proceeded to analyse the mineral composition of the entire colour range that Boyd used and came up with a massive database of minerals that corresponded to his palette - establishing the first component of the work. The second stage was to develop a means of embodying this abstract dataset and this was achieved by sampling the Steinway grand-piano at the Bundanon homestead, note by note. Firstly, we recorded regular keystrokes with conventional microphony. In a second phase, we embedded contact microphones deep within the piano to record the reverberance of the sounding board, 
which resulted in one to two-minute sound files per note.

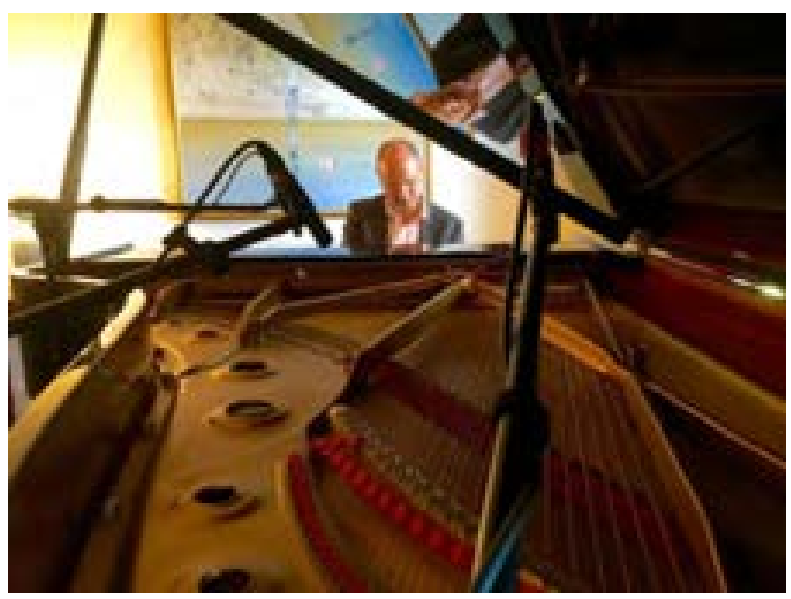

Figure 3. Jon Drummond sampling the Steinway Piano. Image Nigel Helyer.

Working with another colleague, Jon Drummond, who is an expert in data sonification, we created a computer-driven audio-visual system capable of reading the video stream from a camera facing Boyd's unfinished painting Return of the Prodigal Son (c1997). The screen interface displays a highly-magnified colour 'target' area from the painting along with the associated RGB values and a periodic table in which the predominant minerals present in the area of focus are highlighted. The system then translates the stream of mineral and colour data into sound, which is layered in two components. The harmonic chord-like sounds recorded from deep within the piano are assigned to the colour palette and this is overlaid by individual note highlights that illustrate the distribution of the most prominent minerals, the more prevalent an element the greater the number of its assigned note - however the system randomises the note sequences to create randomised trills. As a participant moves the camera across the painting the computer monitor gives feedback on the area of interest, colour ratios and a graphical display of the minerals detected.

The Cultural Theorist Cecelia Cmielewski invokes the presence of Heavy Metal during its debut in the following lyrical extract from the catalogue to the Landscape/Portrait exhibition that documented the When Science meets Art research project.

Winding toward Boyd's studio through the beautiful garden at Bundanon, with flowering azaleas and orange clivias, under the shade of bright-green deciduous amber trees, the chordlike sounds of a piano become increasingly distinguishable. The sounds do not make a discernible melody but, once in the studio, they exert an immediate calming and almost meditative effect.
Visitors are intrigued and delighted by this work. Occasionally a high note pops into the space that jolts the listener into attentiveness. The sound of a painting - Artists have responded to paintings by playing music to them, but not until Heavy Metal have, they composed music from their material composition.

This work is a genuine collaboration between scientist and artist. It is rare for such a collaboration to be a creative conjuncture of both disciplines. Usually one is at the behest of the other. Either the art is used to explain or 'communicate' the science or the science is made too simple by the art. Helyer has a good grasp of many scientific principles and has worked with scientists for over thirty years, the results of which we see in this collaboration.

Heavy Metal is interactive at a complex and conceptual level. The composition of chord-like sounds (recorded from the homestead Steinway) is created by a real-time analysis of the minerals in the colours of an unfinished Boyd painting, Return of the Prodigal Son (c1997). As a video camera is trained onto a section of the canvas, the screen displays the mineral content of the selected colours, in the form of the periodic table. The image and corresponding sound change each time someone selects a new section of the canvas on which to train thecamera. Heavy Metal also brings together two kinds of science: environmental and computational.

The creative leap of the artist is matched with the precise methodology of the scientist. Heavy Metal could have been quite a cold work simply a digital archaeology of a painting. Instead, it is a lively work that uses the warm sounds of the piano and finely calibrated composition to bring the painting into a new space for contemplation. Heavy Metal provides participants with different ways to animate a 'static' painting. It takes some time for viewers/listeners to put together what it is that they are experiencing. The sound is dynamic, based on the elements used in a particular area of Boyd's oil painting.

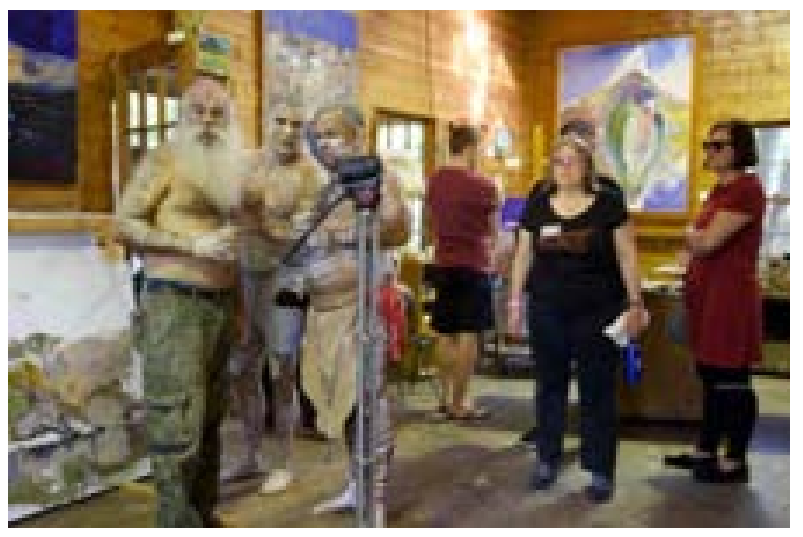

Figure 4. In the studio with Elders from the Wreck Bay community. Image Nigel Helyer 
One little boy of about seven years old knows the periodic table. He is thrilled to be able to 'read' this painting because each sequence on the screen includes a representation of the elements from a particular section of the painting. All of a sudden, he is able to correlate his knowledge of the periodic table with the materials used by the artist. For another visitor, a writer, who does not usually 'get' art, the layers of sound and data provide a way for her to consider the work beyond that of colour and texture. For some of the men visiting from the Wreck Bay community, the data resonates with their use of naturally occurring materials used to paint their bodies for ceremony. Everyone who comes to Siteworks (and there is a large audience) spends time with Heavy Metal. The studio stays open for several hours longer than scheduled, and many visitors come back more than once. As the sounds from the studio close down, the chorus of the frogs in the nearby lake take over in the dark of evening at Bundanon.

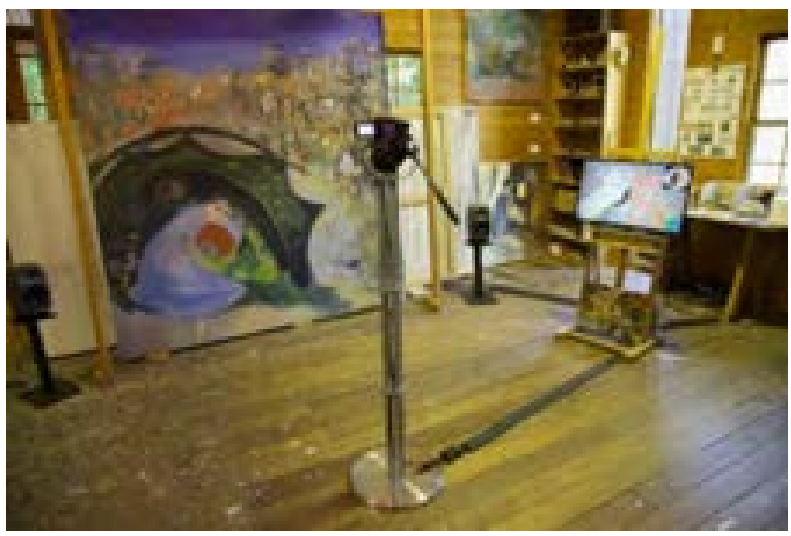

Figure 5. The camera vision system, screen and target painting. Image Nigel Helyer.

\section{CONCLUSION - ART SCIENCE AND THE ENVIRONMENT}

Heavy Metal, draws upon recent research that has sought to develop collaboration between art and science, particularly in the context of technology and the environment. Siân Ede has proposed that there is 'much in contemporary science that can stimulate art's flexible, intuitive and visceral response to the world'. Ede also argues that 'the fragile environment' might well become 'the most crucial matter for the future concerns of both artists and scientists'. Concern for the environment has become a central political and artistic issue in the contemporary world. Recent publications have emphasised the heightened regard for the environment in 'eco-aesthetics', in 'eco art in pursuit of a sustainable planet', in 'art and ecology now' as manifest in 'land art' incorporating landscape, earthworks, environmental art, sculpture, and nature-based installation art. Sean Cubitt has argued that eco-politics is indeed the single largest unifying political discourse of the early $21^{\text {st }}$ century' Cubitt suggests that artworks can voice the contradictions of their period, including the role of technology. It may be demonstrated that 'not all technologies are instrumental, that is, used as instruments for domination over nature'. Media forms and art works may rely on certain technologies to communicate an ecological sensitivity.

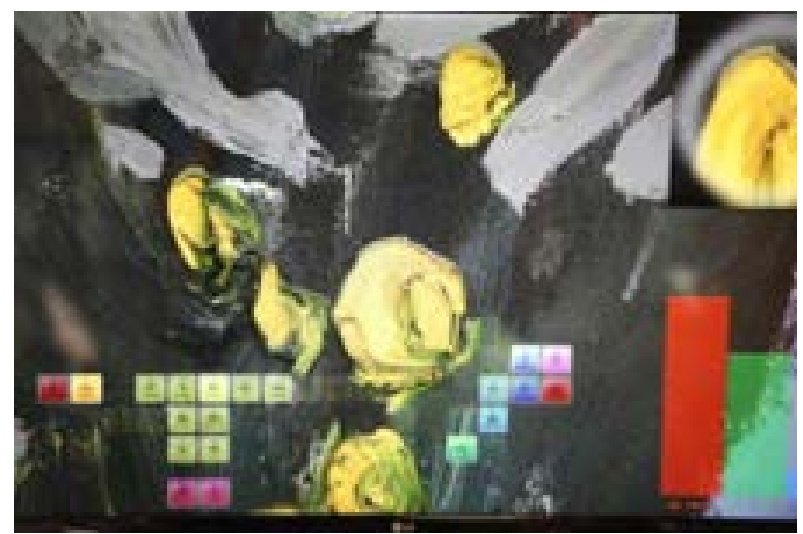

Figure 6. Detail of the Screen showing target area and Periodic Table. Image Nigel Helyer.

\section{ORATORIO FOR A MILLION SOULS}

\subsection{Introduction and context: Oratorio for a Million Souls - Why Bees?}

Why Bees? - There are two main reasons for such interest - firstly Bees, along with other colonial insects (such as termites and ants) display striking social organisation - that since ancient times they have provided powerful metaphors for human social structures. Secondly Bees have become a focus species in the public debate about the environment. Unfortunately, this focus upon the threat to Bees; upon Colony collapse and the subsequent effects on disruptions to food production frequently masks the even wider environmental issues of diminishing Bio-diversity in the face of industrial culture; the mal-practices of agribusiness and planet-wide implications of climate change.

But to return to metaphor - historically the Bee has been and remains a symbol of good (hierarchical) government - with a vast population of obedient workers ruled over by an autocratic Queen. In the English language, we still use the terms such as; As bus $y$ as a $B$ ee and $A$ hi ve of industry as phrases that affirm diligence and application to a task. Until quite recently the reproductive cycle of the Bee was a mystery and the Queen was long regarded by the Christian Church as analogous to the Virgin Mary; until it was discovered that she made a single but prolonged nuptial flight mating with up to eighty males, retaining their sperm for the duration of her long productive life — not such a chaste Lady after all! 


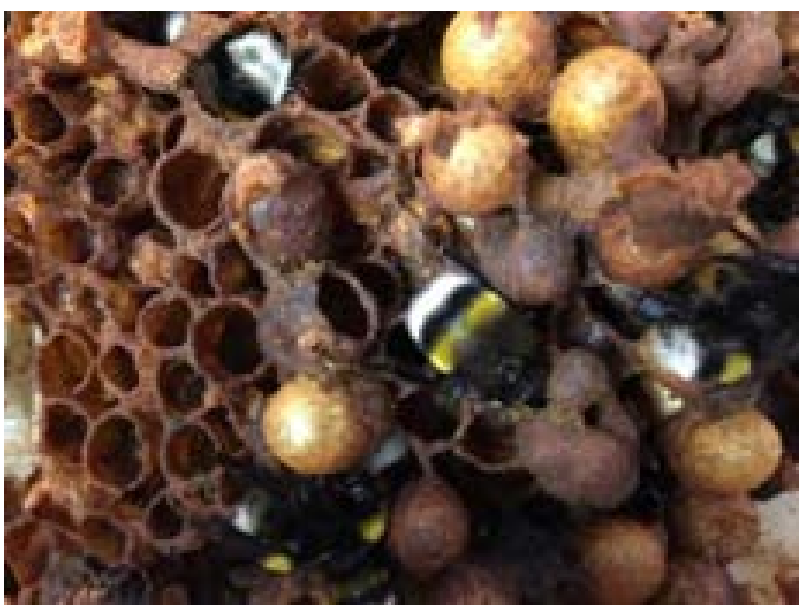

Figure 7. A Bombus Terrestris nest. Image Nigel Helyer.

The workers who make up the vast majority of the hive population, have stood for loyalty, obedience, courage and selflessness and much of their behaviour would seem to support these anthropomorphic metaphors we attach to them perfect role models for Nineteenth Century industrial capital, which like the Monarchy is based upon an autocratic power pyramid.

However, recent research turns this pyramid image upon its head. Consider for a moment the behaviour of bees in Swarming mode. The hive has grown and the colony divides. The outgoing group muster, hanging from a branch somewhere, seeking a new location. The swarm sends out a stream of scouts, often over a period of days, who report back, using methods similar to the bee dance to relay complex qualitative information. Somehow a collective process is engaged, the swarm considers this growing matrix of spatial data and eventually they fly to the most favoured location to begin a new colony. This is not the work of an individual mind, it is a product of parallel processing, a natural neural network, if you like, that has evolved over some 100 million years, the hive as super-organism.

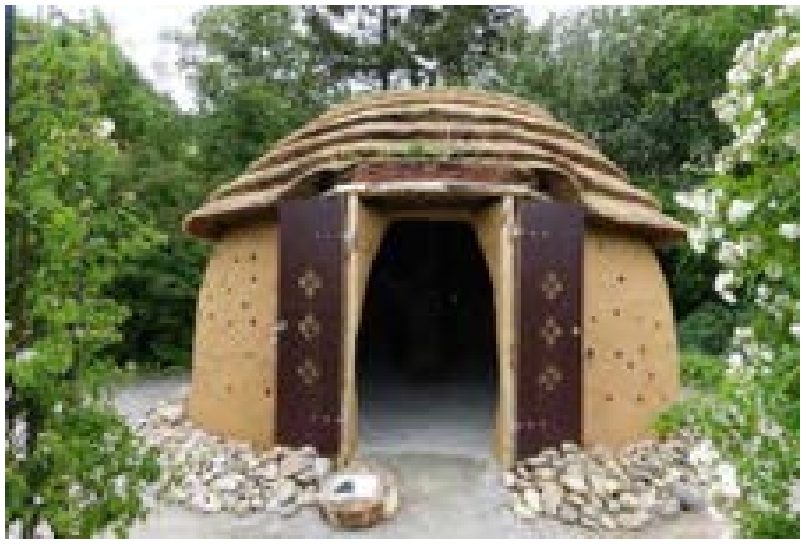

Figure 8. The Oratorio at Buitenpost Gardens. Image Nigel Helyer.

\subsection{European Capital of Culture Leeuwarden 2018}

The Oratorio for a Million Souls ${ }^{\text {vii }}$ was commissioned by the European Capital of Culture, Leeuwarden 2018 (Netherlands). Funded by a European Union Inter-Regional programme the project has been realised in three botanical gardens; Dr Kruidhof in Buitenpost (Netherlands) and in Emden and Oldenburg (Germany) with a working lifecycle of three years. Each site consists of a 'green-architectural' listening space that houses two intelligent bee-hives to produce a fourchannel immersive soundscape in real-time.

The Oratorio for a Million Souls was developed in conjunction with a citizen-science project in which one hundred sensor equipped beehives were distributed to schools in the Netherlands and Germany with the aim of mapping and assessing the pollen load (or floral productivity) of local ecologies. This research is taking place in an environmental context which is commonly referred to as the European "Green Desert" a landscape which at first glance appears to be verdant and productive, but which, in reality is the product of high-intensity mono-culture, industrial agriculture that is effectively eliminating bio-diversity and causing significant reductions in native species of birds and insects.

The concept behind Oratorio is to create a network of bio-acoustic listening spaces that allow visitors to immerse themselves in a world of insect sounds. Visitors are invited to enter the sonic heart of a Bee-city, to suspend their dis-belief and for a moment become a fellow citizen - meditating at the centre of a sonic metropolis; all working; all buzzing - and taking an empathetic leap that we hope permits some serious thoughts about our collective effects on the natural environment.

Physically, each Oratorio is constructed in traditional wattle and daub, intricately hand woven willow branches plastered with a thick layer of Leem viii with a form based upon the shape of a traditional woven straw beehive (a Skepp in English). The structures have a thatched roof reminiscent of the traditional farming buildings of the region, and this in turn supports a green-roof garden - sown with Bee attracting flowers - a sort of Bee-Helipad! The hemispherical interior of the Oratorios provides a special acoustic, sharing characteristics with the domed roofs of Cathedrals and Mosques where sound loses its directionality and forms an omni-directional soundcloud.

Each of these green architectures contains two sensor-hives furnished with colonies of Bombus Terrestris $^{i x}$ the common white tailed Bumble Bee.

We employ high definition miniature DPA microphones $^{x}$ in the Bee entry ports that are 
capable of recording the sounds of individuals as they arrive or depart the nest; as well as a set of large scale piezo contact microphones within the nest area that record the collective activity of the hive. These two streams of live audio are mixed with sonifications of Bee entry and exit data which are rendered as short arpeggios of piano notes; rising upon exit and descending upon arrival, and which become more complex with the frequency of Bee activity.

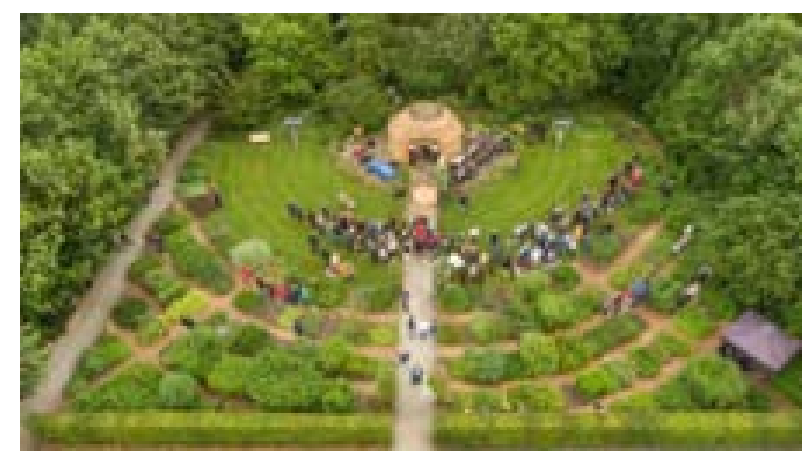

Figure 9. The Oratorio concert. Image de Kruidhof Buitenpost.

The arpeggios provide visitors inside the Oratorio with a direct sonic index of the intensity of Bee flight activity as this can only be seen from the exterior of the structure. In addition, hive temperature is rendered as a series of sustained tones that rise or fall as the temperature within each hive varies. All of these outputs from the two hives are combined into a four-channel live soundscape which is augmented by a screen displaying real-time data of bee activity and data sonification.

The province of Fryslân in the Netherlands and the adjacent area of Ost-Friesland in Germany boast many talented town Brass Ensembles and we were also commissioned to create a series of musical scores derived from the sounds and data sets recorded in the Oratorios, that could link each of the sites. By analysing the harmonic range of hive recordings, we derived a tonal palette that we quantised into an equal tempered scale. Likewise, by analysing the Bee entry and exit data from a range of the citizen science hives we established a temporal or rhythmic framework as well as developing an overall compositional structure based upon the diurnal activity of a hive (which is related to light and temperature).

The province of Fryslân in the Netherlands and the adjacent area of Ost-Friesland in Germany boast many talented town Brass Ensembles and we were also commissioned to create a series of musical scores derived from the sounds and data sets recorded in the Oratorios, that could link each of the sites. By analysing the harmonic range of hive recordings, we derived a tonal palette that we quantised into an equal tempered scale. Likewise, by analysing the Bee entry and exit data from a range of the citizen science hives we established a temporal or rhythmic framework as well as developing an overall compositional structure based upon the diurnal activity of a hive (which is related to light and temperature).

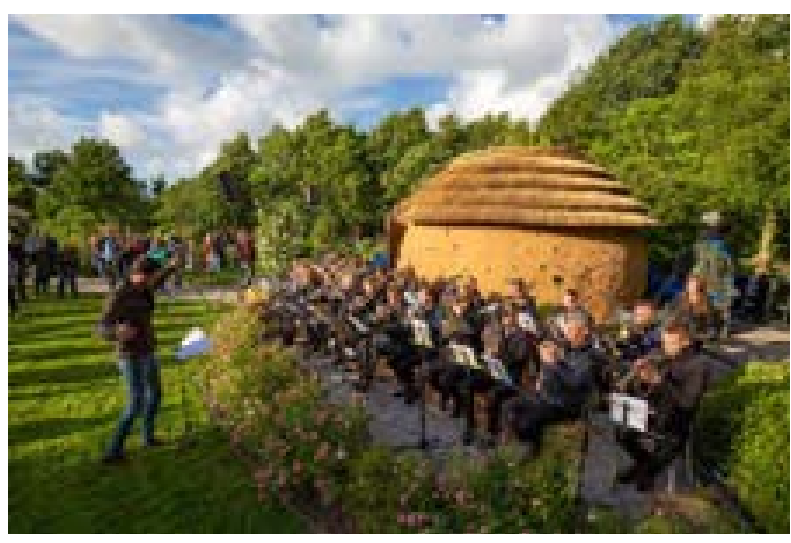

Figure 10. B_Rhapsodie in performance. Image Nigel Helyer.

We created a musical event in which the three Brass ensembles located in Buitenpost, Emden and Oldenburg, collaborated to simultaneously play a multi-part score via a satellite Video and Audio link-up - a task not without technical or aesthetic challenges, but which thanks to the skill of the technical crew and a deal of good luck worked perfectly!

\subsection{Creative Challenges - The Oratorios}

Working with living biological systems is always complex and unpredictable - Bees especially so in terms of handling nests of flying (and potentially stinging) insects who do not like being disturbed! This made the work of testing and mounting microphones inside the hives a physical challenge. Acoustically the project also had to develop solutions to prevent feedback within the sound architecture, which contained the two hives as live acoustic sources. The highly sensitive DPA microphones embedded in the Bee entry/exit ports provide an extremely detailed audio stream of flight and crawling sounds and as they are located on the building exterior are not affected by the interior soundscape. In contrast, our early experiments with conventional microphones placed inside the hive boxes were a complete failure due to persistent feedback.

As the interior of the nests produce a range of relatively quiet, crawling, peeping and contact events conventional studio microphones require considerable amplification - the hive boxes 
themselves resonate with the sound energy from the four-channel speaker array setting up a feedback loop. A solution was provided by wiring together two large format Piezo discs and mounting them onto a small sounding board which was placed onto the upper surface of the nest enclosure. This extremely low-tech approximation of a contact microphone delivered a remarkably good sound fidelity and eliminated the problems of airborne audio feedback.

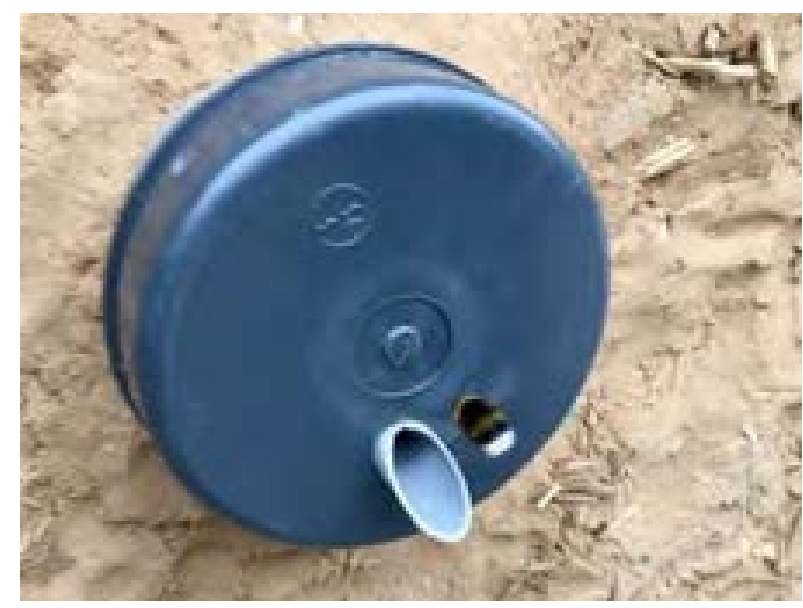

Figure 11. A Bumblebee about to enter the Oratorio. Image Nigel Helyer.

\subsection{Creative Challenges - The Musical Scores}

From the perspective of traditional musical aesthetics both the temporal and pitch structures of the Bee audio and Bee-data derived composition are unconventional. Whilst the three Brass Ensembles were musically extremely competent there was some initial resistance as our scores certainly did not resemble their normal repertoire the $B$-Rhapsodie score is certainly not an imagistic representation of the life of a hive (a la Flight of the Bumble-Bee) but rather an indexical translation of that life and activity.

The debut of the composition B-Rhapsodie took place during June 2018 across the three sites. The collaborative rendition of the score by three geographically distant Brass Ensembles was a logistically difficult operation. We had to carefully synchronise the musicians and deal with the inevitable time lag in satellite transmission. Each site could see and hear the other two distant ensembles - and somehow, despite cloud breaks of cold European summer rain trickling into and English Horns the concert was a success!

\subsection{Legacy}

In line with the objectives of the European Capital of Culture, works included in the programme are designed to have a legacy that benefits and extends the cultural life of the region. The Oratorio for a Million Souls project and its linked citizen science collaboration have an ongoing life in terms of research; public advocacy (for the preservation and growth of Bio-diversity) and in the very palpable form of the three Oratorios in their respective botanical gardens which are designed to function for at least three years. Each botanical garden is free to incorporate the Oratorio into its visitor and education programme giving a new and extended meaning to the authors original intentions.

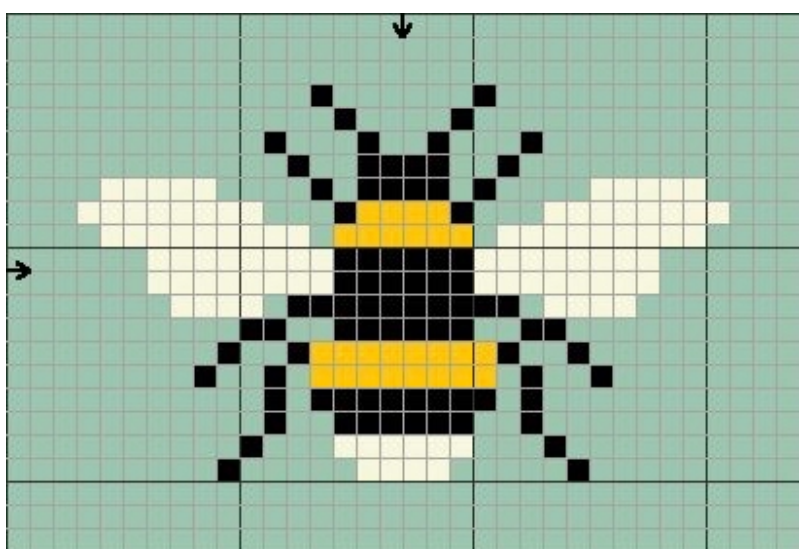

Figure 12. Lorem ipsum.

i Heavy Metal <http://www.sonicobjects.com/index. php/projects/more/heavy_metal>

ii The Bundanon Trust N $S W$ Australia https://bundanon. com

iii Arthur Boyd; One of the first Australian painters to be widely recognised internationally, specifically in the United Kingdom as one of the Antipodeans.

iv Culturescape <http://www.culturescape.net.au>

v Helyer.N. 2017 Landscape/Portrait catalogue. Macquarie University Art Gallery ISBN: 978-1-74138-450-5 2017

vi To generate the data programmed into Heavy Metal, we used a hand-held X-ray fluorescence spectrometer.

vii Cmielewski. C. 2017 Landscape/Portrait catalogue. Macquarie University Art Gallery ISBN: 978-1-74138450-5 2017 pages 32 33.

viii Ede.S. 2005 Art and Science, I.B.Tauris. ISBN: 9781850435839

ix Oratorio for a Million souls archive <http://www.sonic objects.com/index.php/projects/more/oratorio_for_a_milli on_souls>

$x$ Leem - a mixture of clay, sand and chopped straw.

$x i$ The bee nests we used were supplied commercially. In Holland much of the agriculture takes place in vast green-houses and the pollination is carried out by Bumble bees who are 'buzz' pollinators - using their wing muscles to vibrate pollen from the flower. xii DPA 4060 omnidirectional lavalier microphones 